\title{
MODELACIÓN DE ÁREAS INUNDABLES EN UN SECTOR MINERO DE LA CUENCA QUEBRADA EL ARRAYÁN - REGIÓN DE COQUIMBO - CHILE.
}

\author{
MODELING OF FLOODABLE AREAS IN A MINING SECTOR OF THE ARRAYAN \\ BASIN - COQUIMBO REGION - CHILE.
}

\author{
Keyla Manuela Alencar da Silva Alves ${ }^{(*)}$, Constanza Belén Contreras Franco y María Carolina Parodi \\ Dávila
}

Facultad de Ingeniería. Universidad Tecnológica Metropolitana. Santiago, Chile.

${ }^{(*)}$ e_mail: keyla.dasilva@utem.cl

\begin{abstract}
RESUMEN
La cuenca hidrográfica Quebrada del Arrayán, se encuentra ubicada en la región de Coquimbo, al norte de Chile. Es una zona reconocida por la intensa actividad minera de extracción de cobre y oro, y por los inúmeros tranques de relaves instalados en los alrededores de la zona urbana a oeste de la cuenca. Existen 108 tranques de relaves instalados en el sector oeste de la cuenca, la mayoría de ellos en estado de abandono o inactividad. El sector oeste de la cuenca es un área susceptible a inundaciones, destacando las ocurridas en 1997, 2015, 2017 y 2020. El clima semiárido con régimen de precipitaciones concentradas en pocos días, combinado con sectores de depresión en montaña, como la pampa ondulada y los cordones transversales, son factores que contribuyen con la susceptibilidad de inundación en el área de estudio. La dinámica hídrica en zonas con concentración de numerosos tranques de relaves abandonados puede representar un agravamiento potencial del riesgo, considerando el arrastre de metales pesados y la contaminación del agua y suelo. Como resultado, por medio de modelación matemática en SIG se identificó que $63 \%$ de los tranques de relaves abandonados se encuentran instalados en zonas inundables y cercanos a sectores poblados.
\end{abstract}

Palabras clave: inundaciones, modelos, SIG, tranques de relave, minería.

\begin{abstract}
The Arrayan basin is in the Coquimbo region of northern Chile. It is an area of intense mining activity for copper and gold extraction, and for the numerous tailings dams installed in the urban area west of the basin. There are 108 tailings dams in the western sector of the basin, most of them abandoned or inactive. The western sector of the basin is an area susceptible to flooding, with floods occurring in 1997, 2015, 2017 and 2020. The semi-arid climate with rainfall concentrated in a few days, combined with mountain depression sectors, such as the undulating plain to the west of the basin, are factors that contribute to the susceptibility to flooding in the study area. Water dynamics in areas with a concentration of abandoned tailings dams may represent a potential aggravation of the risk, considering the entrainment of heavy metals and water and soil contamination. As a result, through mathematical modeling in GIS, it was identified that $63 \%$ of the abandoned tailings dams are in flood zones and close to populated areas.
\end{abstract}

Keywords: floods, modeling, GIS, tailings dams, mining. 


\section{INTRODUCCIÓN}

La industria minera en Chile produce 5.82 millones de toneladas métricas de cobre fino; sin embargo, su extracción genera una tasa de producción de relaves de 530 millones de metros cúbicos $\left(\mathrm{m}^{3}\right)$ al año (SERNAGEOMIN, 2019). Los tranques son la técnica más común de depositación de residuo sólido (sedimentos) minero en Chile, y pueden encontrase en tres diferentes tipos de estado de funcionamiento: activo, inactivo o abandonado.

Los tranques son inherentemente inestables y suelen concentrar grandes cantidades de substancias tóxicas (Kossoff et al., 2014). Conforme al último catastro del Servicio Nacional de Geología y Minería de Chile (SERNAGEOMIN) actualizado al 10 de agosto de 2020, Chile cuenta con 757 tranques de relaves en total, donde 112 son activos, 5 se encuentran en construcción, 173 están abandonados y 467 inactivos.

El norte de Chile es la región donde concentran la mayor cantidad de tranques de relaves del país. En lo cual se destaca la región de Coquimbo con la mayor concentración de tranques, con 244 inactivos y 106 abandonados, según el Catastro Nacional de Relaves (SERNAGEOMIN, 2020).

En la cuenca Quebrada del Arrayán, ubicada en la región de Coquimbo, específicamente en el sector urbanizado de dicha cuenca, donde se ubica la Comuna de Andacollo, es el sector a nivel nacional donde se concentran la mayor cantidad de tranques de relaves. Existen 108 tranques de relaves, entre ellos 35 se encuentran abandonados, 5 activos, 68 inactivos y los demás sin identificar su estado actual.

Además, de acuerdo con la base de datos geoquímica de SERNAGEOMIN, los relaves abandonados e inactivos en Andacollo superan el límite máximo de concentración en cobre, arsénico, plomo, cadmio y níquel según la norma internacional canadiense (Canadian Soil Quality Guidelines for the Protection of Environmental and Human Health, 2018).

Otro aspecto relevante respecto al riesgo ambiental e impacto a la salud de las personas que representa la existencia de estos tranques de relaves en lugares poblados son las características físicas topográficas de donde están instalados los tranques. Según la CEPAL, 57\% de los tranques de relaves a nivel nacional se encuentran instalados en zonas susceptibles a remociones en masa e inundaciones (Silva y Souza, 2020). En este contexto, se enfatiza las característica hidrológicas y geomorfológicas de la cuenca quebrada del Arrayán, principalmente en el sector donde se concentran los tranques. Dicha cuenca se encuentra ubicada en la pampa ondulada de los valles transversales. Los tranques están instalados en la zona de depresión de la cuenca donde ya han ocurrido inundaciones, cuales se destacan los eventos ocurridos en 1997, 2015, 2017 y 2020, que resultaron en el colapso de algunos tranques de relaves (Miralles, 2013; Rojas et al., 2014; Mardones, 2019, Pacheco et al., 2020).

Los estudios de zonificación de la susceptibilidad y del riesgo asociados a los impactos ocasionados por eventos naturales adquiere un potencial peligro cuando estos eventos se desarrollan en zonas donde hay acumulación de residuos peligros, tales como residuos mineros. Por lo tanto, caracterizar las áreas susceptibles a la inundación y correlacionarla con la ubicación de los tranques de relaves contribuirá con la definición de las áreas de riesgo de contaminación por esparcimientos de los desechos tóxicos.

\section{OBJETIVOS}

En este contexto, considerando el potencial peligro de ocurrencia de inundaciones y consecuentemente el colapso de los tranques de relaves y la dispersión de los residuos mineros presentes en el sector oeste de la cuenca Quebrada del Arrayán, este estudio tiene como objetivo identificar las zonas susceptibles a inundaciones por medio del modelo Geomorphic Flood Area (GFA) del software Qgis 2.18.3, y a partir de resultado obtenido del GFA, correlacionarlo con la ubicación de los tranques de relaves y zonificar las áreas de riesgo en el sector urbanizado (zonas pobladas) de la cuenca.

\section{METODOLOGÍA}

La metodología de este estudio consiste en tres etapas: 1. Clasificación del estado de funcionamiento de los tranques de relaves existentes en el área de estudio, utilizando la base de datos del Catastro Nacional de Relaves 2020 del SERNAGEOMIN. 2. Zonificación de áreas inundables por medio de la ejecución del modelo Geomorphic Flood Area (GFA) integrado al software Qgis 2.18. 3. Mapeo de las zonas de riesgo basándose en los criterios implementados por la Guía de Evaluación de Riesgos Ambientales (MMA, 
2010) y el Plan Regulador Intercomunal de la región de Coquimbo (2019).

\section{Área de estudio}

La Cuenca Quebrada del Arrayán forma parte de la gran cuenca del río Elqui y se encuentra en la región de Coquimbo, Chile. Cuenta con un área de 557.72 $\mathrm{km}^{2}$ y un perímetro de $134.64 \mathrm{~km}$ (Figura 1). Su superficie se extiende sobre las comunas de Andacollo y Vicuña, siendo esta última el punto de desembocadura de la quebrada.

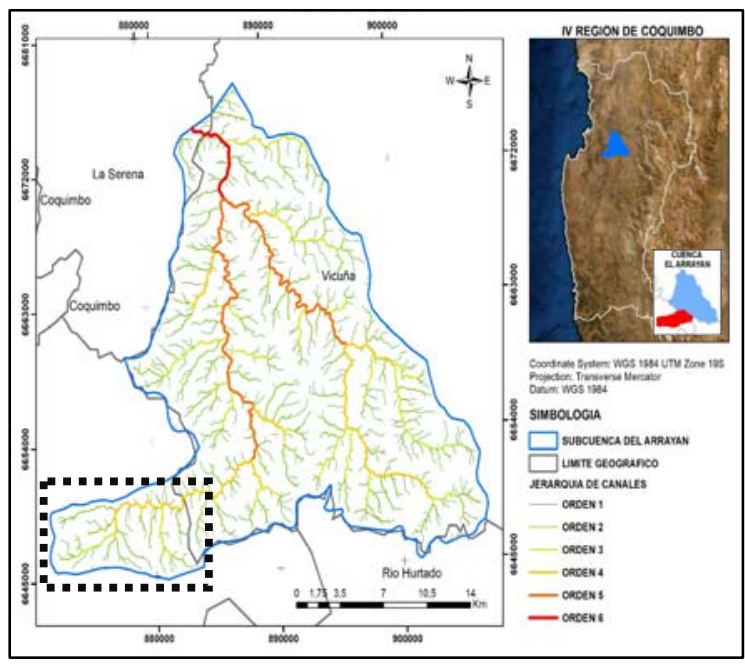

Figura 1. Ubicación y jerarquía del drenaje de la cuenca

Quebrada del Arrayán. En destaque el área de estudio.

El área de estudio se encuentra rodeadas de grandes montañas, ya que forma parte de las últimas ramificaciones de la cordillera de los Andes en el Norte Chico de Chile. Posee vegetación tipo matorral xerófila. La zona cuenta con un clima de carácter estepa cálida los que se caracteriza por ser un clima de transición entre los desiertos cálidos y los climas semiáridos (Squeo et al., 2001; Bascuñán, 2010; Cortés et al., 2012).

Las precipitaciones son concentradas e intensas, considerando las características climáticas locales, en el contexto de la aridez regional. Se intensifican bajo la influencia del fenómeno de El Niño, registrando un promedio de precipitaciones de 130 $\mathrm{mm}$ anuales en el sector oeste de la cuenca. No obstante, el total de precipitaciones anuales están concentradas en 1 o 2 días de precipitaciones (Salas et al., 2012, Scaff, 2012). Donde fueron registradas ocurrencias de inundaciones al oeste de la cuenca durante los años 1997, 2015, 2017 y 2020, siendo este un sector urbanizado y minero.

\section{Clasificación del estado de funcionamiento de los tranques de relaves}

Desde del Catastro Nacional de relaves obtenidos de la base de datos del Sernageomin, se creó un mapa representativo de la distribución de los tranques de relaves instalados en la cuenca. Fueron identificados 108 tranques de relaves mineros, de los cuales 35 en estado de abandonados, 5 activos y 68 inactivos. Todos ubicados en el sector oeste de la cuenca, siendo este mismo sector el más urbanizado de la cuenca, donde se localiza la comuna de Andacollo. La delimitación del área urbana y minera en este sector de la cuenca fue realizada por medio de fotointerpretación de imágenes satelitales Google Earth (Figura 2).

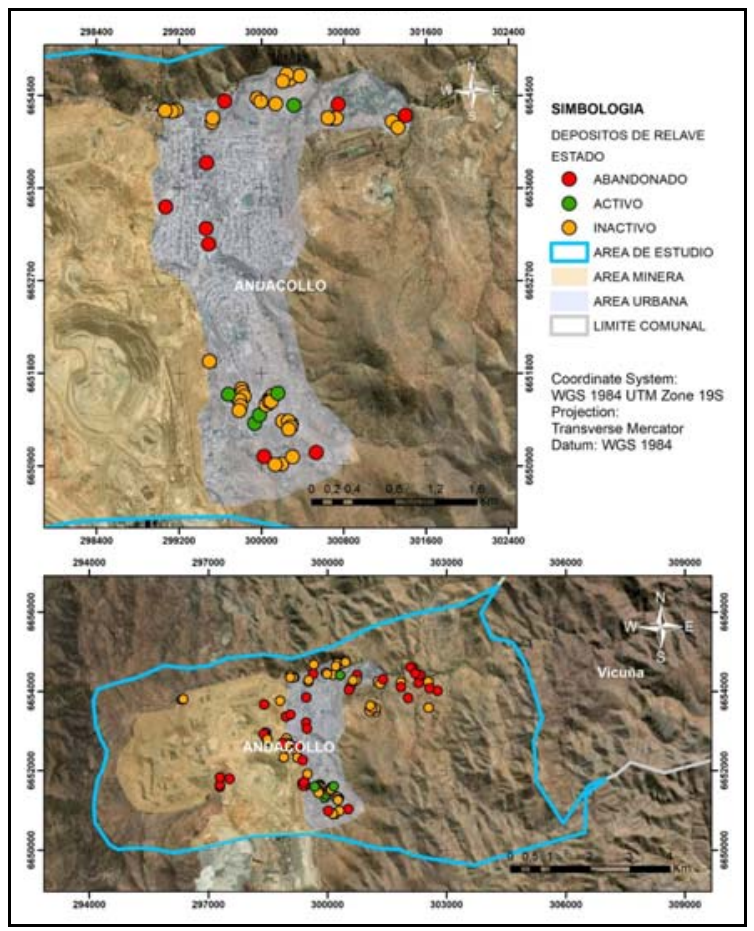

Figura 2. Distribución espacial de la ubicación de los tranques de relaves, clasificados según estado de funcionamiento.

En Chile existen normas legislativas que regulan la estabilidad física y química de los tranques de relaves, incluyendo factores del proceso de creación y desarrollo, desde su apertura, operación, cierre y post - cierre, hasta el tipo de diseño y construcción. El artículo $3^{\circ}$ de la ley 2055/2011 apunta que la estabilidad física corresponde a una forma de seguridad dirigida en la resistencia y la disminución de fuerzas que desestabilicen los tranques u obras de una faena minera, empleando medidas para evitar 
fallas, colapsos o remoción. Mientras que el reglamento de la ley de cierre de faenas e instalaciones mineras apunta que la estabilidad química es una situación de control en agua, aire y suelo, de las características químicas que se encuentran en los materiales en tranques $u$ obras de una faena minera, para de esta forma evitar, prevenir o eliminar reacciones químicas y evitar el contacto del agua con residuos que se encuentren en obras y tranques mineros.

No obstante, dichas normas presentes en la ley $20551 / 2011$ tiene restricciones y no es retroactiva a su fecha de publicación. Es decir, los criterios presentes presentados en ley 20551/2011 solo son aplicadas a los tranques de relaves que estén en estado activo o inactivo (a partir del año de publicación de la ley). De esta forma, todos los tranques de relaves que fueron inactivos anterior a 2011 no están regulados por la ley mencionada anteriormente. Siendo estos pasivos ambientales un potencial riesgo para población circundante $\mathrm{y}$ al medio ambiente.

Los tranques de relaves abandonados no se encuentran identificados por ninguna normativa vigente, por lo tanto, no están bajo ningún parámetro de seguridad que garantice su estabilidad física o química, lo que implica que aquellos que se encuentren en estado de abandonado presentan un mayor riesgo para la salud de las personas y los ecosistemas cercanos.

Por lo tanto, en base a la clasificación del estado de funcionamiento de los tranques de relaves, se establece su grado de peligrosidad, según su estado físico, siendo: los tranques de relaves Activos de baja peligrosidad, los inactivos de mediana peligrosidad y los abandonados de alta peligrosidad (MMA, 2012).

Zonificación de áreas inundables por medio de la

La herramienta Geomorphic Flood Area permite derivar el mapa de susceptibilidad de inundación de una cuenca combinando información geomorfológica extraída del DEM, junto con información de riesgo de inundación derivada de mapas de inundación preexistentes, generalmente disponibles para porciones limitadas de una cuenca en los planes reguladores comunales.

Este método permite clasificar los puntos dentro de una cuenca en dos grupos: áreas propensas a inundaciones y áreas no propensas a inundaciones, utilizando un referente binario lineal clasificador basado en el índice de inundación geomórfica (GFI) (Samela et al., 2017; Faridani et al., 2020, Alencar da Silva et al., 2021).

Según Samela et al. (2020), el GFA posee un logaritmo natural entre la relación entre una profundidad de agua variable $h_{r}$ y la diferencia de elevación $\mathrm{H}$, cuya ecuación se describe a continuación:

$\mathrm{GFA}=\operatorname{Ln}\left(\mathrm{h}_{\mathrm{r}} / \mathrm{H}\right)$

Donde $h_{r}$ representa la profundidad de agua de la red fluvial más cercano a un punto cualquiera de la cuenca y $\mathrm{H}$ corresponde a la diferencia de altura entre ambos puntos mencionados anteriormente. Procedimiento iterativo para cada punto de la cuenca.

$\mathrm{h}_{\mathrm{r}} \approx \mathrm{A}_{\mathrm{r}}^{\mathrm{n}}$

Donde $h_{r}$ es la profundidad del agua (m), $A_{r}\left(\mathrm{~km}^{2}\right)$ es el área contribuyente calculada en el punto más cercano del río conectada al punto más bajo del cauce, y $n$ es el exponente (sin dimensiones). (Samela et al., 2018).

El índice está basado en parámetros morfométricos y topográficos generales tales como: (A) representación de cuenca fluvial; (B) vista en sección transversal del canal y la llanura de inundación. Área máxima precipitable (C) (Figura 3).

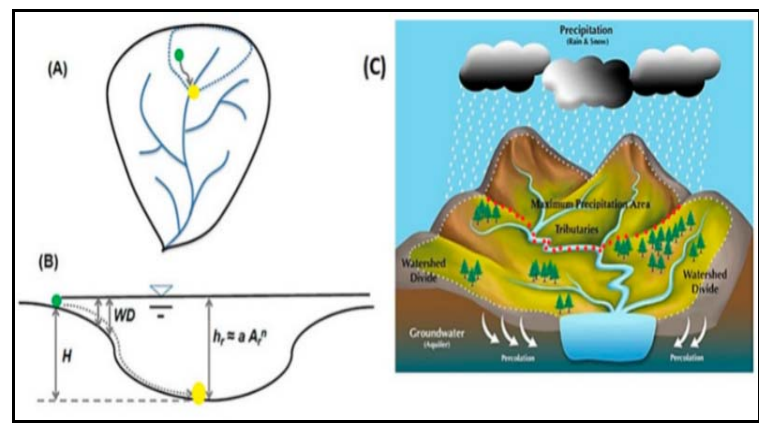

Figura 3. Descripción esquemática de los parámetros utilizados para derivar el Índice de Inundación Geomórfica. Fuente: Faridani et al., 2020.

\section{Mapeo de las zonas de riesgo en el sector urbano minero}

La definición de las áreas de riesgo fue determinada a partir de la intersección del resultado del modelo 
GFA, la ubicación de los tranques de relaves abandonados y las manzanas urbanas de la comuna de Andacollo.

Los criterios implementados fueron basados en los establecidos por la Guía de Evaluación de Riesgos Ambientales (MMA, 2010) y Plan Regulador Intercomunal de la región de Coquimbo (2019), donde detallan los criterios para los distintos riesgos que se identifican en la región de Coquimbo, entre ellos se encuentran el peligro de inundaciones costeras asociadas a maremotos, los cuales corresponden a:

- Zonas de Susceptibilidad Muy Alta: sectores ubicados bajo la cota $8 \mathrm{~m}$.

- Zonas de Susceptibilidad Alta: sectores ubicados entre las cotas 8 y $12 \mathrm{~m}$.

- Zonas de Susceptibilidad Moderada: sectores ubicados entre las cotas 12 y $16 \mathrm{~m}$.

Donde para efecto de esta investigación se adaptó lo anterior a inundaciones producidas por crecidas que corresponden al evento prominente en la zona de estudio. La adaptación consta de un promedio entre las cotas que bordean las áreas propensas a inundaciones que entran en contacto con el área urbana, obteniendo una cota promedio de $1064 \mathrm{~m}$, a la cual se le consideraron los 12 metros más para definir el área de riesgo moderado, resultando en las siguientes categorías:

- Áreas con riesgo muy alto: Resultado obtenido del GFA, es decir, áreas propensas a inundaciones.

- Áreas con riesgo moderado: Cotas entre $1064 \mathrm{~m}$ y $1076 \mathrm{~m}$.

- Áreas sin ningún riesgo: Lo restante sobre la cota $1076 \mathrm{~m}$.

\section{RESULTADOS Y DISCUSIÓN}

\section{Simulación de las áreas inundables}

El modelo GFA simula las áreas inundables a partir de las características topográficas de la cuenca. De esta forma, el modelo entrega un resultado único rasterizado del área inundable. La cuenca Quebrada del Arrayán se encuentra en la zona de los valles transversales. Pero a pesar de las altas altitudes, el sector oeste de la cuenca, que corresponde al sector estudiado, geomorfológicamente es formado por una zona de planicie, definida como pampa ondulada, que está ubicada entre dos cordones transversales cordilleranos.

También fue caracterizado el suelo según su uso urbano y minero, para definir el alcance de las áreas inundables en estos sectores, donde se obtuvo los siguientes resultados de mensura de inundación (Figura 4):

- Un total de $9.73 \mathrm{~km}^{2}$ de área de inundación en el sector oeste de la cuenca en estudio.

- Del total del área de minería un $1.84 \mathrm{~km}^{2}$ corresponde a zona de inundación.

- Del total de área urbana un $1.67 \mathrm{~km}^{2}$ corresponde a área propensa a inundación.

\section{Resultado de intersección entre las zonas susceptibles a inundación e inventario de tranques de relaves.}

Obtenida el área susceptible a inundación, se realizó una intersección con la capa georreferenciada de los tranques de relaves, la información fue según estado de funcionamiento actual activo, inactivo $y$ abandonados (Figura 5).

Este resultado corrobora con lo evidenciado en terreno durante las visitas ocurridas en marzo de 2020 y con los registros de inundaciones en los años 1997, 2015, 2017 y 2020. Los registros erosivos identificados durante las visitas a terreno comprueban la exposición de los tranques a procesos erosivos hídricos, y la susceptibilidad de transporte del material sedimentario, que presenta baja cohesión. La erosión hídrica proporciona perdida de material y transporte de estos tranques de relaves. En particular, es importante enfatizar que el material transportado son sedimentos mineralizados que poseen altas concentraciones de metales pesados, altamente peligros a la salud de las personas. 


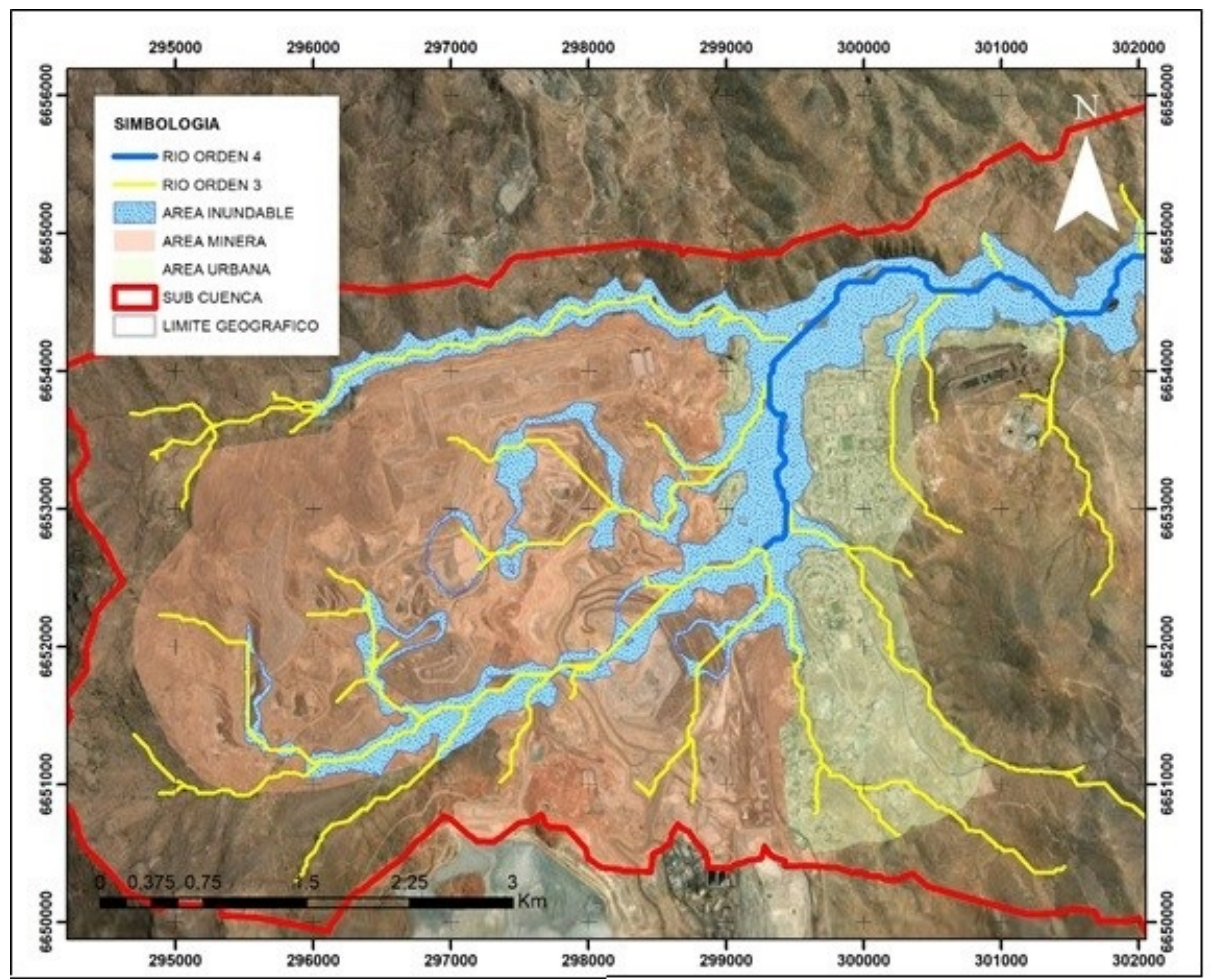

Figura 4. Áreas inundables resultantes de la modelación GFA y el uso de suelo correspondiente al área de estudio.

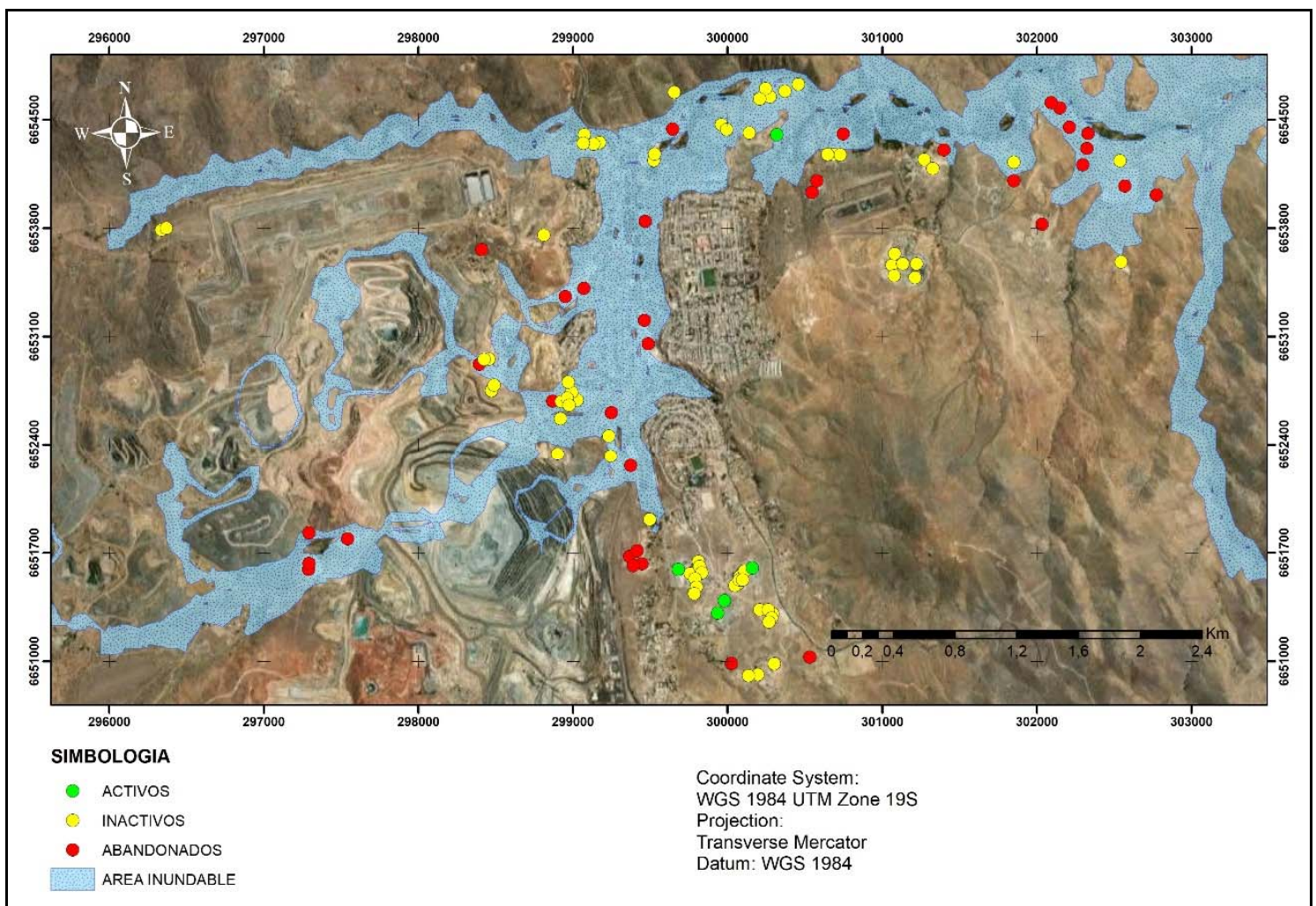

Figura 5. Intersección de las áreas inundables con la ubicación de los tranques de relaves activos, inactivos y abandonados instalados en el sector oeste de la cuenca Quebrada del Arrayán. 
La intersección de los resultados presentados anteriormente demuestra que $63 \%$ de total de tranques de relaves abandonados se encuentra instalados en las áreas inundables. Esto representa un peligro ya que estos tranques no están bajo ninguna normativa y están compuestos por valores sobre las normativas internaciones respecto a la concentración total de metales pesados. Los demás porcentajes en relación con la ubicación de los tranques se presentan en la siguiente Tabla 1:

Tabla 1: Porcentaje de tranques de relaves en área propensa inundación. (Fuente: Elaboración de las autoras.)

\begin{tabular}{|l|l|l|l|}
\hline \multicolumn{1}{|c}{$\begin{array}{c}\text { Estado de } \\
\text { funcionamiento }\end{array}$} & $\begin{array}{c}\mathrm{N}^{\circ} \text { de } \\
\text { tranques } \\
\text { de relaves }\end{array}$ & $\begin{array}{c}\text { Área } \\
\text { inundable }\end{array}$ & $\begin{array}{c}\text { de } \\
\text { tranques } \\
\text { de } \\
\text { relaves } \\
\text { en área } \\
\text { inundable }\end{array}$ \\
\hline activos & 5 & 1 & 20 \\
\hline inactivos & 68 & 26 & 38 \\
\hline abandonados & 35 & 22 & 63 \\
\hline total & 108 & 49 & 45 \\
\hline
\end{tabular}

Del total de 108 tranques de relaves ubicados en la cuenca, 49 de estos se encuentran dentro del área propensa a inundaciones, lo que corresponde a un $45 \%$ del total, donde:

- De los 5 tranques de relaves ubicados en la comuna de Andacollo en estado activo, 1 se encuentra dentro del área propensa a inundación, lo que es equivalente a un $20 \%$.

- De los 68 tranques de relaves ubicados en la comuna de Andacollo en estado inactivo, 26 se encuentra dentro del área propensa a inundación, lo que es equivalente a un $38 \%$.

- De los 35 tranques de relaves ubicados en la comuna de Andacollo en estado abandonado, 22 se encuentra dentro del área propensa a inundación, lo que es equivalente a un $63 \%$.

\section{Zonificación de las áreas de riesgo}

En este estudio el concepto de riesgo hidrológico no se marca como el único factor que proporciona peligro a la población. Dada las características de uso del suelo presentados anteriormente, la actividad minera y la gran cantidad de tranques instalados en el área de estudio, proporciona un aumento potencial del riesgo. Principalmente considerando la de los 35 tranques de relaves existentes 22 se encuentran instalados en zonas inundables.

El estado de deterioro avanzado de muchos de los relaves abandonados se refleja en la inestabilidad estructural de ellos, y consecuentemente llevando al colapso parcial y/o total de ellos. De esta forma aumentando el radio de contaminación, por medio del transporte de los sedimentos mineralizados (Alencar da Silva et al., 2021).

La zonificación de riesgo en este estudio consistió en cruzar los datos espaciales de dos amenazas: áreas inundables $\mathrm{y}$ tranques de relaves abandonados, con el objetivo de destacar los sectores que representa mayor riesgo en función del mayor número de relaves abandonados por manzana urbanas y los rangos de nivel de la inundación en función de la altura. Para esto fueron aplicados los criterios basándose en la Guía de Evaluación de Riesgos Ambientales (MMA, 2010) y el Plan Regulador Intercomunal de la región de Coquimbo (2019). En el Plan Regulador de Coquimbo se detallan los parámetros aplicados para definir los distintos riesgos que se identifican en la región de Coquimbo. Entre ellos se encuentran el peligro de inundaciones costeras, los cuales corresponden a las categorías presentadas en la metodología.

Donde para efecto de este estudio se adaptó lo anterior a inundaciones en zonas de valles transversales que corresponden al evento prominente en la zona de estudio. La adaptación consta de un promedio entre las cotas que bordean las áreas propensas a inundaciones que entran en contacto con el área urbana, obteniendo una cota promedio de $1064 \mathrm{~m}$, a la cual se le consideraron los 12 metros más para definir el área de riesgo moderado, lo cual resulta de la siguiente manera (Figura 6):

- Áreas con riesgo muy alto: Resultado obtenido del GFA, es decir, áreas propensas a inundaciones.

- Áreas con riesgo moderado: Cotas entre $1064 \mathrm{~m}$ y $1076 \mathrm{~m}$.

- Áreas sin ningún riesgo: Lo restante sobre la cota $1076 \mathrm{~m}$. 


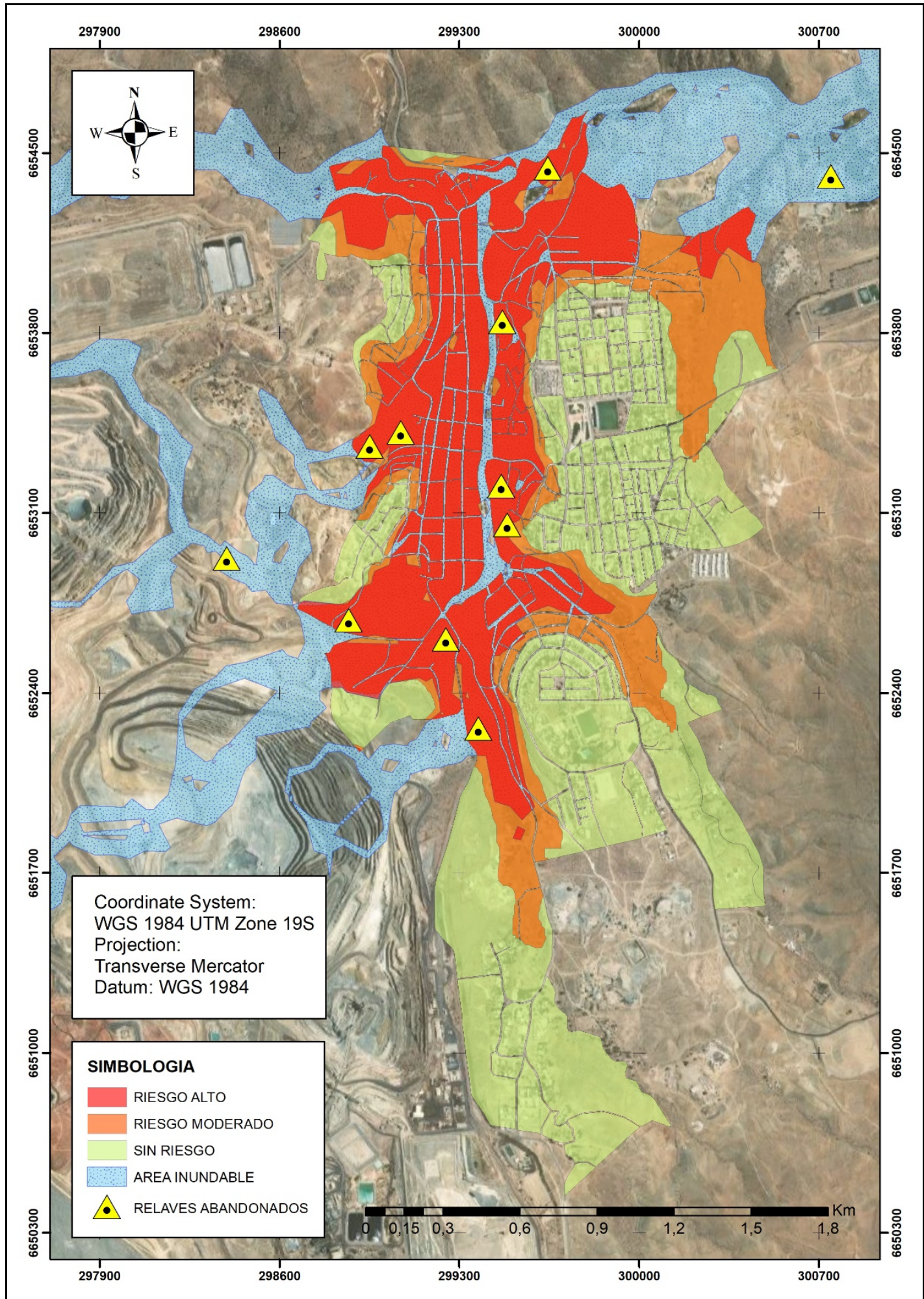

Figura 6. Zonificacion de áreas de riesgo según la interseccion de áreas inundables, tranques de relaves abandonados y sectores poblados de la cuenca Quebrada de Arrayán. 
Las áreas inundables resultantes del modelo GFA, que además coincidieran con la presencia de tranques de relaves $y$ áreas pobladas fueron definidas como las áreas de alto riesgo (Zonas en color rojo). Las zonas de riesgo moderado fueron el resultado de la adaptación realizada en base a los criterios establecidos por el Plan Regulador Intercomunal de la región de Coquimbo y las manzanas urbanas que representan las zonas pobladas. En la zona de riesgo moderado no se encuentra instalados ninguno de los tranques de relaves (zonas en color naranja). Por último, la zona que no presenta ningún riesgo son las que se encuentra sobre la cota $1076 \mathrm{~m}$. El riesgo es nulo en esta zona porque no existen tranques de relaves instalados, ni tampoco fue identificada como área inundable según los resultados del modelo GFA.

\section{CONCLUSIONES}

Este estudio corroboró con la identificación de la ubicación inadecuada de los tranques de relaves en la cuenca Quebrada del Arrayán, cruzando datos de la modelación de áreas inundables e inventario de tranques de relaves georreferenciados, instalados en zonas pabladas de la cuenca.

El modelo GFA es un modelo de base geomórfica, define las áreas inundables solamente a partir de las características topográficas del terreno. Para cumplimiento del objetivo propuesto para este estudio, el modelo GFA entregó resultados que posibilitaron identificar si los tranques de relaves existentes estaban instalados en una zona inundable. No obstante, para estudios específicos de la dinámica hidrológica de las inundaciones, el modelo GFA no es compatible, ya que solamente entrega como resultado el área de inundación.

Considerando los resultados que indican que $63 \%$ de los tranques de relaves abandonados se encuentran instalados en zonas inundables, que también son zonas pobladas. Se concluye que el riesgo fundamental en el área de estudio es la posibilidad de colapso y/o transporte del material de los tranques de relaves durante un evento hidrometereológico tal como una inundación. El riesgo de inundaciones en el área de estudios se intensifica una vez que en la zona potencialmente susceptible a la ocurrencia de dicho evento se encuentra instalados depósitos de residuos mineros que están compuestos mayoritariamente por metales pesados.
El uso de los modelos numéricos combinados con herramientas SIG son recursos de muy bajo costo y que entregan excelentes resultados para la gestión de riesgo, que puedan venir a colaborar con planes de mitigación y políticas públicas a nivel comunal.

\section{AGRADECIMIENTOS}

Iniciativa financiada por Agencia Nacional de Investigación y Desarrollo (ANID), por medio del "Primer Concurso IDeA de I+D del FONDO DE FOMENTO AL DESARROLLO CIENTÍFICO Y TECNOLÓGICO, FONDEF/CONYCIT, 2018 Fondef ID18I10189” y apoyada por la Municipalidad de Andacollo y por la Universidad Tecnológica Metropolitana (UTEM) de Chile. Se agradece también al Laboratorio de Suelos y Medio Ambiente, dependiente del Departamento de Industria, Facultad de Ingeniería de la UTEM y al Consejo Nacional de Investigación y Técnicas (CONICET - ARGENTINA) por medio de la beca postdoctoral Latinoamérica 2020, por el apoyo en la ejecución del estudio.

\section{REFERENCIAS}

Alencar da Silva, A. K. M., Parodi, D. M. C., Zimmermann, E. (2021). Diagnóstico y Zonificación de Áreas Susceptibles a Inundaciones en la Comuna De Andacollo - Región De Coquimbo - Chile. Revista Contexto Geográfico, 6(11), 44-55.

Bascuñán Walker, F. (2010). Crecimiento urbano y balance de $\mathrm{CO} 2$ en la cuenca del río Elqui (Chile). Bosque (Valdivia), 31(2), 109-116.

Canadian Council of Ministers of the Environment CCME, 2018. Canadian Soil Quality Guidelines for the Protection of Environmental and Human Health.

Cortés, A. E., Oyarzún, R., Kretschmer, N., Chaves, H., Soto, G., Soto, M., ... \& Maturana, H. (2012). Aplicación del Índice de Sustentabilidad de Cuencas en la cuenca del río Elqui, Centro-Norte de Chile. Obras y proyectos, 12, 57-69.

Faridani, F., Bakhtiari, S., Faridhosseini, A., Gibson, M. J., Farmani, R., \& Lasaponara, R. (2020). Estimating Flood Characteristics Using Geomorphologic Flood Index with Regards to Rainfall Intensity-Duration-Frequency-Area Curves and CADDIES-2D Model in Three Iranian Basins. Sustainability, 12(18), 7371. 
Kossoff, D., Dubbin, W. E., Alfredsson, M., Edwards, S. J., Macklin, M. G., Hudson-Edwards, K. A. (2014). Mine tailings dams: characteristics, failure, environmental impacts, and remediation. Applied Geochemistry, 51, 229-245.

Ley $\mathrm{N}^{\circ}$ 20551/2011 Regulación del Cierre De Faenas e Instalaciones Mineras. 28-10-2011. Ministerio de Minería. ID norma 1032158.

Mardones Morales, P. (2019). Análisis estadístico de precipitaciones desencadenantes de flujos de detritos en la Región de Coquimbo. Disponible en http://repositorio.uchile.cl/handle/2250/170764.

Miralles González, C. (2013). Evaluación de los factores que controlan la geoquímica de sedimentos fluviales de la cuenca del río Elqui, IV Región de Coquimbo, Chile. Disponible en http://repositorio.uchile.cl/handle/2250/115370.

MMA. Ministerio del Medio Ambiente, P. (2010). Guía de evaluación de riesgos ambientales.

MMA. Ministerio del Medio Ambiente (2012). Guía Metodológica para la Gestión de Suelos con Potencial Presencia de Contaminantes.

Pacheco, P. R., Parodi, M. C., Mera, E. M., Salini, G. A. (2020). Variables meteorológicas y niveles de concentración de material particulado de $10 \mu \mathrm{m}$ en Andacollo, Chile: un estudio de dispersión y entropías. Información tecnológica, 31(6), 171 182.

Plan Regulador Intercomunal de la región de Coquimbo, G. R. I. R. (2019, April). Resolución-1 10-Abr-2019 Ministerio del Interior y Seguridad Pública; Gobierno Regional Iv Región De Coquimbo (Biblioteca del Congreso| Ley Chile). Biblioteca del Congreso Nacional de Chile.

Rojas, O., Mardones, M., Arumí, J. L., \& Aguayo, M. (2014). Una revisión de inundaciones fluviales en Chile, período 1574-2012: causas, recurrencia y efectos geográficos. Revista de Geografía Norte Grande, (57), 177-192.

Salas, S., Jiménez, E., Montaña, E., GarayFlühmann, R., Gauthier, D., \& Díaz, H. P. (2012). Vulnerabilidad al cambio climático, desafíos para la adaptación en las cuencas de Elqui y Mendoza. La Serena, Chile. Editora del Norte.

Samela, C., Troy, T. J., Manfreda, S. (2017). Geomorphic classifiers for flood-prone areas delineation for data-scarce environments. Advances in water resources, 102, 13-28.

Samela, C., Albano, R., Sole, A., \& Manfreda, S. (2018). A GIS tool for cost-effective delineation of flood-prone areas. Computers, Environment and Urban Systems, 70, 43-52.
Samela, C., Persiano, S., Bagli, S., Luzzi, V., Mazzoli, P., Humer, G., Castellarin, A. (2020). Safer_RAIN: a DEM-based hierarchical filling-\&Spilling algorithm for pluvial flood hazard assessment and mapping across large urban areas. Water, 12(6), 1514.

Scaff, F. M. (2012). Interpretación hidrometeorológica de los gradientes orográficos de precipitación en el Valle del Elqui. Disponible en http://repositorio.uchile.cl/handle/2250/112074.

SERNAGEOMIN. Servicio Nacional de Geología y Minería, 2019. Anuario de la Minería de Chile, https://www.sernageomin.cl/pdf/anuario_2019_act 100720.pdf

SERNAGEOMIN. Servicio Nacional De Geología y Minería, 2020. Catastro Nacional Depósito de relaves. Disponible desde internet en: https:/www.sernageomin.cl/deposito-de-relaves/ (con acceso el 13/10/2021).

Silva, M., Souza, G. (2020). Metodologías para el uso de factores de emisión: material particulado en depósitos de relaves abandonados, serie Medio Ambiente y Desarrollo, No 170 LC/TS.2020/92), Santiago, Comisión Económica para América Latina y el Caribe (CEPAL).

Squeo, F. A., Arancio, G., Cavieres, L. A. (2001). Sitios prioritarios para la conservación de la flora nativa con riesgos de extinción en la IV Región de Coquimbo, Chile. Libro rojo de la flora nativa y de los Sitios Prioritarios para su Conservación: Región de Coquimbo, 171-193.

\section{Tipo de Publicación: ARTíCULO.}

\section{Trabajo recibido el 08/11/2021 y aprobado para su publicación el 05/12/2021.}

\section{COMO CITAR}

Alencar da Silva, A. K. M.; Contreras Franco, C. B. y Parodi, D. M. C. (2021). Modelación de áreas inundables en un sector minero de la cuenca Quebrada El Arrayán - Región de Coquimbo Chile.. Cuadernos del CURIHAM, 27, 59-68. DOI: https://doi.org/10.35305/curiham.v27i.170

Este es un artículo de acceso abierto bajo licencia: Creative Commons Atribución - No Comercial Compartir Igual 4.0 Internacional (CC BY-NC-SA 4.0) (https://creativecommons.org/licenses/by-ncsa/4.0/deed.es) 